\title{
Delayed Diagnosed Stage 1, 2 Distractive Flexion Injury of the Cervical Spine
}

\author{
Taek-Soo Jeon'1, Han Chang', Young-Bok Kim¹, Byung-Hak Oh², Sang-Bum Kim², \\ Tae-Seok Nam¹, Ji-Wan Kim¹, Kun-Bo Park' ${ }^{1}$, Hyun-Wook Chung ${ }^{1}$ \\ 'Spine Center, Haeundae Paik Hospital, Inje University, Busan, Korea \\ ${ }^{2}$ Department of Orthopaedic Surgery, Konyang University College of Medicine, Daejeon, Korea
}

\begin{abstract}
Study Design: Retrospective study.
Purpose: To examine the clinical and radiologic characteristics of patients with stage 1 and 2 distractive flexion injury according to Allen's classification and who were not diagnosed immediately after injury, and to analyze the outcomes of surgical treatments.

Overview of Literature: For the diagnosis of stage 1 and 2 distractive flexion injury in the lower cervical spine, attention should be paid when performing radiographs as well as when interpreting the radiographs.

Methods: The study was conducted on 10 patients (group 1) with stage 1 or 2 distractive flexion injury and who were not diagnosed immediately after injury from January 2003 to January 2009. The control group (group 2), 16 distractive flexion injury patients who were diagnosed immediately were selected. The simple radiographs, the degree of soft tissue swelling and the magnetic resonance imaging findings of the two groups were compared, and the clinical and radiologic results were examined.

Results: The degree of the prevertebral soft tissue swelling of group 1 was lower in group 1, and it was statistically significant $(p=0.046$ ). The fusion was achieved in all cases $(100 \%)$ in group 1 , however, re-displacement as well as the loss of reduction occurred in one case, despite of delayed fusion and good clinical result. In group 2, bone fusion was achieved in 15 cases of 16 cases (94\%).

Conclusions: For the diagnosis of stage 1 and 2 distractive flexion injury in the lower cervical spine, it is desirable to perform computed tomography if diagnosis is not clear. Even if the diagnosis is delayed, stage 1 and 2 distractive flexion injury could be readily reduced by traction, and the treatment outcomes are considered to be comparable to those of the patients diagnosed immediately after injury.
\end{abstract}

Key Words: Cervical spine, Distractive flexion injury, Unilateral facet fracture-dislocation, Anterior cevical discectomy and fusion, Delayed diagnosis

\section{Introduction}

Unilateral facet fracture-dislocation of the lower cervical spine is caused by distractive flexion injury, and these fracture-dislocations correspond to stage 1 (unilateral subluxation) or stage 2 (unilateral dislocation) of the Allen system [1] that classifies them according to the level of injury. The

Received Jul 21, 2010; 1st Revised Nov 11, 2010; Accepted Nov 18, 2010

Corresponding author: Sang-Bum Kim, MD

Department of Orthopedic Surgery, Konyang University College of Medicine, 685 Gasuwon-dong, Seo-gu, Daejeon 302-718, Korea Tel: +82-42-600-6937, Fax: +82-42-545-2373, E-mail: sbkim@kyuh.co.kr

* The abstract of this study was presented as a lecture at the 26th fall conference of the Korean Spinal Surgeons in 2009. 
stage 1 injury has the characteristics that the posterior ligament complex and the posterior facet capsules may be ruptured at diverse levels, but the intervertebral disc is not injured, the flexion causes subluxation and the interspinous interval is increased. In stage 2, unilateral facet dislocation occurs and the characteristic feature of this is the anterior displacement of the upper vertebral body. Stage 1 or 2 distractive flexion injury occurs preferentially in the lower cervical spine, and the level of the external force at the time of injury is not relatively large. Thus, the clinical symptoms are meager and neurologic deficit hardly occurs, so the diagnosis is delayed in some cases [2-6]. In the clinical setting, distractive flexion injury is seen relatively frequently, but there are only rare studies on the treatment outcomes of the cases with a delayed diagnosis of distractive flexion injury [2-4,7]. Particularly, there have been no studies on stage 1 and 2 distractive flexion injury that consisted of mostly cases with a delayed diagnosis. Therefore, we divided the patients with unilateral facet fracture subluxation or dislocation in the lower cervical spine caused by stage 1 or 2 distractive flexion injury to the group that was not detected immediately after injury and so the diagnosis was delayed, and the group that was diagnosed immediately after injury. We examined their clinical and radiological characteristics, and we analyzed the outcomes of surgical treatment. The causes of a delayed diagnosis were analyzed, and the differences in the treatments and outcomes between the two groups were examined.

\section{Materials and Methods}

From January 2003 to April 2009, among the 11 patients with stage 1 or 2 distractive flexion injury in the lower cervical spine according to Allen's classification and who were not diagnosed immediately after injury, we excluded 1 patient with laryngeal cancer for whom the anterior approach could not be performed. So, a total of 10 cases were assigned as the group 1 and we then conducted a retrospective study on them. The control group patients were 16 stage 1 or 2 distractive flexion injury patients who were diagnosed immediately after injury and they underwent surgery during the same period and they were assigned as group 2. We examined the differences of age, gender, the mechanism of injury, and radiological as well as clinical characteristics of the two groups. In addition, we analyzed the cause of a delayed diagnosis in group 1, and the differences in the treatment methods and outcomes between the two groups.

\section{The patient group and treatment methods}

The mean age of the patients in group 1 was 52.1 years (range, 38 to 68 years), and that of group 2 was 46.6 years (range, 28 to 72 years). In regard to the gender distribution, there were 8 males and 2 females in group 1 . There were 12 male patients and 4 female patients in group 2. In group 1, the interval from injury to diagnosis was on average 22.7 days (range, 2 to 51 days) and the interval from injury to surgery in group 1 was on average 27.6 days (range, 2 to 51 days). In group 2, this was on average 5.1 days (range, 1 to 10 days). As for the cause of injury, there were 7 cases of motor vehicle accident, 1 case of blunt trauma, 1 case of falling down and 1 case of slip-down in group 1. In group 2, there were 12 cases of motor vehicle accident, 1 case of blunt injury, 1 case of falling down and 2 cases of slipdown. The mean follow-up period of group 1 was 12.9 months (range, 6 to 29 months), and that for group 2 was 13.2 months (range, 6 to 39 months). The age, gender, the cause of injury and the follow-up observation period of the two groups were not statistically different (Table 1).

At the time of diagnosis, persistent posterior neck pain and the limitation of motion of the neck were the findings detected in almost all the patients, and radiculopathy was seen in all the cases of group 1 and in 9 cases of group 2 $(60 \%)$. One case developed neurologic deficit in group 1 $(10 \%)$, and it was grade D of Frankel's grade. There were 4 cases of neurological deficit in group $2(25 \%)$ : there were 2 cases of grade A and 1 case each of grade B and grade C. The injury level of group 1 was the 5th-6th cervical vertebra in 6 cases and the 6th-7th cervical vertebra in 4 cases. In group 2 , the injury area was the 3rd-4th cervical vertebra in 1 case, the 4th-5th cervical vertebra in 3 cases, the 5th-6th cervical vertebra in 9 cases and the 6th-7th cervical vertebra in 3 cases. With excluding the 1 patient who underwent surgery on the day after diagnosis and 1 case strongly refused skeletal traction, reduction was attempted for 8 patients of group 1 and for all the patients of group 2, and magnetic resonance imaging (MRI) was performed on all the patients.

As for surgical treatments, in all the cases, anterior cervical discectomy according to the left-sided Smith-Robinson approach and interbody fusion using autogenous iliac tricortical strut bone or a cage filled with cancellous bone (Solis ${ }^{\circledR}$, Stryker Spine, South Allendale, NJ, USA) were performed, 
while skeletal traction was maintained, and fixated with metal plate. After surgery, the patients wore Philadelphia braces for 6-8 weeks.

\section{Evaluation}

As markers of radiological evaluation, the retropharyngeal space at the inferior border of the 3rd cervical vertebral body was measured on the plain lateral X-ray images, and the difference between the two groups was assessed. The degree of bone fusion at the final follow-ups was assessed by the application of Bridwell' s classification (Table 2) [8], and this was compared between the 2 groups; the incidence of the associated traumatic intervertebral disc herniation was examined on the magnetic resonance imaging.

The severity of the postoperative neck pain, the neurologic status and the improvement of radiculopathy were examined and compared for determining the clinical results. In addition, the operation time and the incidence of complications were examined. The collected data was analyzed by independent $t$-tests and Fisher's exact test. The SPSS ver. 15.0 (SPSS Inc., Chicago, IL, USA) was used for all the sta-

Table 1. Profile of the cases

\begin{tabular}{|c|c|c|c|c|c|c|c|}
\hline Case & Sex & Age (yr) & Injury cause & $\begin{array}{l}\text { Injury to Dx. } \\
\text { interval (day) }\end{array}$ & $\begin{array}{l}\text { Injury to op. } \\
\text { interval (day) }\end{array}$ & Injury level & Frankel grade \\
\hline \multicolumn{8}{|c|}{ Group I } \\
\hline 1 & $\mathrm{~F}$ & 48 & Fall & 51 & 57 & C5-6 & $\mathrm{E}$ \\
\hline 2 & M & 38 & MVA & 15 & 17 & C5-6 & $\mathrm{E}$ \\
\hline 3 & M & 59 & MVA & 24 & 26 & C6-7 & $\mathrm{E}$ \\
\hline 4 & M & 44 & MVA & 43 & 48 & C6-7 & $\mathrm{E}$ \\
\hline 5 & M & 67 & MVA & 21 & 27 & C6-7 & $\mathrm{E}$ \\
\hline 6 & M & 38 & MVA & 2 & 4 & C5-6 & $\mathrm{D}$ \\
\hline 7 & M & 56 & Slip down & 12 & 20 & C5-6 & $\mathrm{E}$ \\
\hline 8 & M & 59 & Blunt & 9 & 17 & C6-7 & $\mathrm{E}$ \\
\hline 9 & $\mathrm{~F}$ & 68 & MVA & 3 & 11 & C5-6 & $\mathrm{E}$ \\
\hline 10 & M & 44 & MVA & 47 & 49 & C5-6 & $\mathrm{E}$ \\
\hline \multicolumn{8}{|c|}{ Group II } \\
\hline 1 & $\mathrm{~F}$ & 40 & MVA & - & 8 & C5-6 & $\mathrm{E}$ \\
\hline 2 & M & 40 & Blunt & - & 1 & C5-6 & $\mathrm{E}$ \\
\hline 3 & M & 59 & MVA & - & 6 & $\mathrm{C} 4-5$ & $\mathrm{E}$ \\
\hline 4 & M & 44 & MVA & - & 5 & $\mathrm{C} 4-5$ & A \\
\hline 5 & $\mathrm{~F}$ & 45 & MVA & - & 1 & C5-6 & B \\
\hline 6 & M & 72 & Fall & - & 7 & C5-6 & $\mathrm{E}$ \\
\hline 7 & $\mathrm{~F}$ & 59 & MVA & - & 10 & C5-6 & $\mathrm{E}$ \\
\hline 8 & M & 43 & Fall & - & 5 & C3-4 & $\mathrm{E}$ \\
\hline 9 & $\mathrm{~F}$ & 28 & MVA & - & 10 & C5-6 & $\mathrm{E}$ \\
\hline 10 & M & 43 & MVA & - & 5 & C6-7 & $\mathrm{E}$ \\
\hline 11 & M & 33 & MVA & - & 4 & C5-6 & $\mathrm{E}$ \\
\hline 12 & M & 39 & MVA & - & 4 & C5-6 & $\mathrm{E}$ \\
\hline 13 & M & 55 & MVA & - & 7 & C4-5 & $\mathrm{C}$ \\
\hline 14 & M & 48 & MVA & - & 5 & C6-7 & $\mathrm{E}$ \\
\hline 15 & M & 31 & MVA & -- & 3 & C5-6 & $\mathrm{E}$ \\
\hline 16 & M & 66 & Slip Down & & 1 & C6-7 & A \\
\hline
\end{tabular}

F: Female, M: Male, MVA: Motor vehicle accident, Dx.: Daignosis, op.: Operation.

Table 2. Bridwell's anterior fusion grading

\begin{tabular}{ll}
\hline \hline Grade I & Fused with complete remodeling and trabeculae \\
Grade II & Graft intact but not fully incorporated, no radiolucencies \\
Grade III & Graft intact but with definite radiolucency \\
Grade IV & Definitely not fused with resorption of the graft \pm collapse \\
\hline
\end{tabular}


tistics. $p$-values lower than 0.05 were considered to be significant.

\section{Results}

The cause of a delayed diagnosis for 2 cases was that radiologic evaluation was not performed because of unstable vital signs due to associated injury at the time of injury or a concomitant urgent operation. It was impossible to assess the injury levels of 5 cases due to inappropriate radiographs (Fig. 1), and in 3 cases the injury area could be assessed by radiography, but this was overlooked because of the carelessness of the doctors.

In group 1, reduction could be achieved in all cases with attempted skeletal traction. In group 2, reduction could be achieved prior to surgery for all the cases. For 2 cases of group 1 that reduction was not performed prior to surgery, reduction was performed by traction in the operation room under general anesthesia. So reduction could be achieved in all cases, and surgery was performed by the anterior approach.

The degree of soft tissue swelling at the inferior border of the 3rd cervical vertebral body was assessed by measuring the distance of the retropharyngeal space. The degree of group 1 was average $5.25 \pm 1.02 \mathrm{~mm}$ and that of group 2 was $7.49 \pm 2.88 \mathrm{~mm}$. The degree of swelling was lower in the group 1 and this was statistically significant $(p=0.046)$. Two cases of group 1 and 7 cases of group 2 were associated with the herniation of an intervertebral disc as detected by MRI, and this was more frequent in the group 2 . Nonetheless, statistical significance was not shown ( $p=$ 0.229 ).

The operation time of group 1 was on average $103 \mathrm{~min}$ utes (range, 73 to 155 minutes), that of group 2 was on average 124 minutes (range, 105 to 185 minutes) and it was slightly longer in the group 2 . Nevertheless, a statistical difference was not detected $(p=0.205)$. Bone fusion was achieved in all the cases of group 1. According to Bridwell' s classification [8], there were 8 cases of grade I and 2 cases of grade II. In group 2, there were 14 cases of grade I and 1 case of grade II, and 15 cases achieved osseous fusion. There was 1 case of pseudoarthrosis corresponding to grade IV. In 1 case of group 1, the loss of reduction developed 2 weeks after surgery, nevertheless, clinical pain was absent
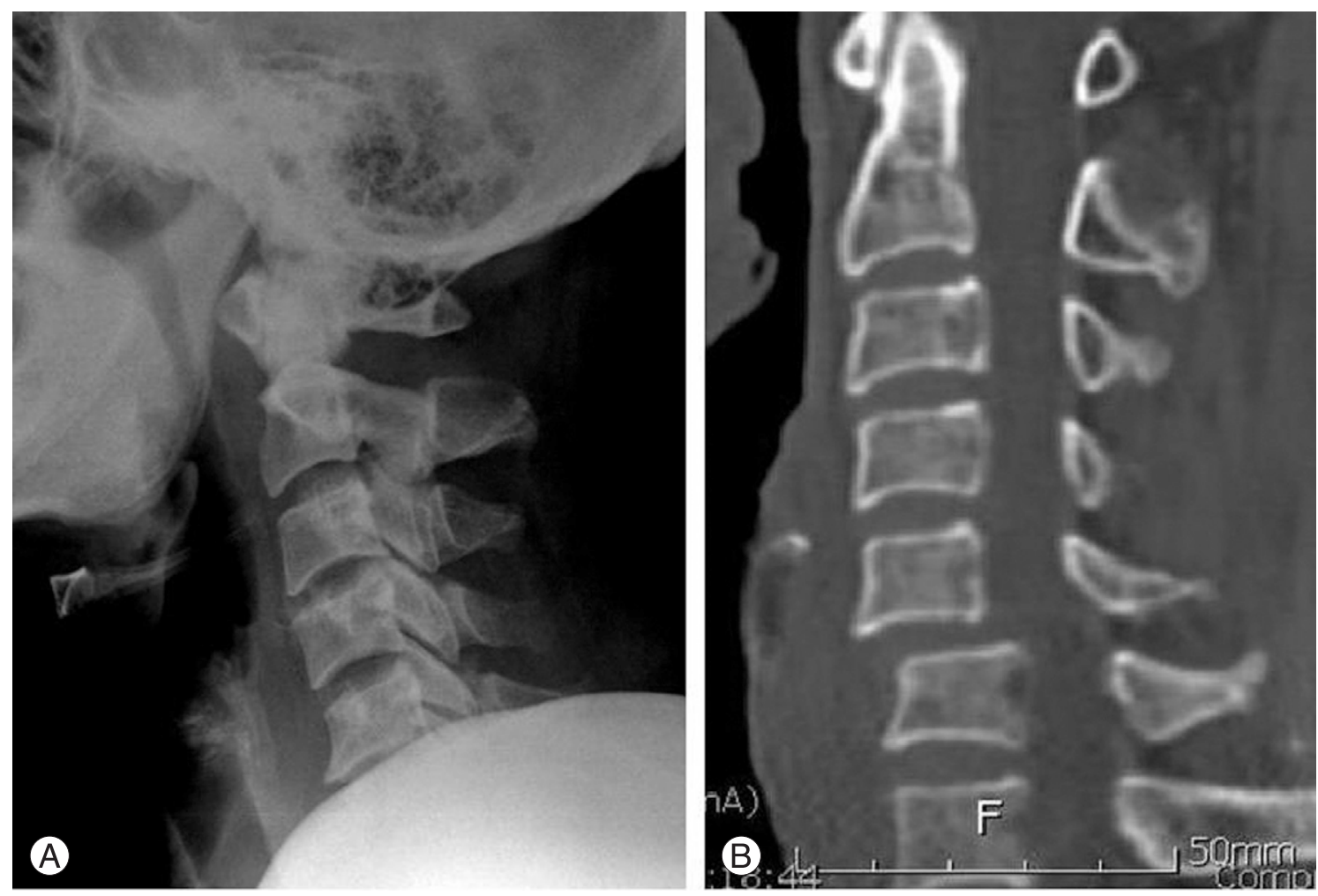

Fig. 1. (A) The lateral radiograph only shows the upper five cervical vertebrae with the retropharyngeal space increased to $6.3 \mathrm{~mm}$. (B) The computed tomography scan clearly demonstrates the dislocation at C5-6 on the sagittal reconstruction image. 

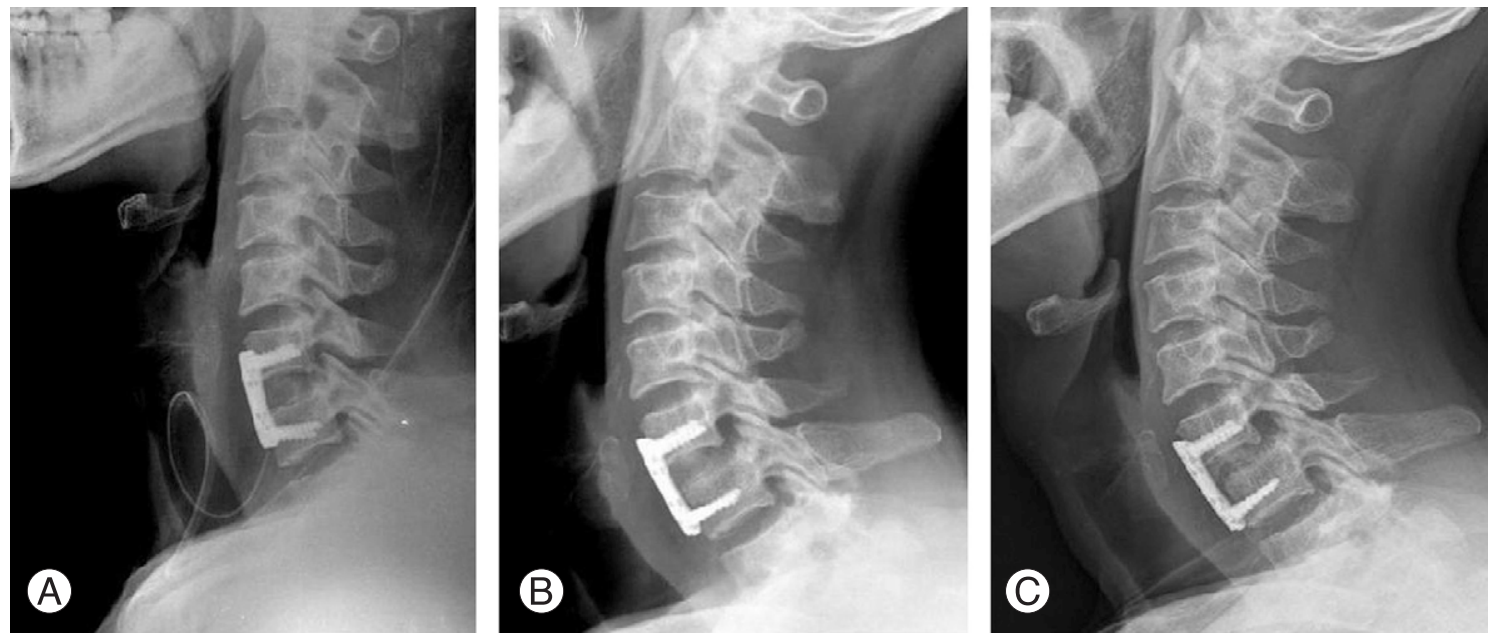

Fig. 2. (A) The immediate postoperative lateral cervical radiograph demonstrates the restoration of the normal sagittal alignment. (B) Displacement has occurred at 2 weeks postoperatively. (C) There is no evidence of further displacement 4 months after the surgery. The radiograph shows a grade II fusion according to Bridwell' s anterior fusion grading.

and neurologic symptoms were not shown, and so only follow-up observation was performed. Bone fusion was confirmed at the final follow-up observation in this case (Fig. 2). One case of group 2 showed non-fusion, the progression of the loosening of screws, the displacement of the graft bone and resorption, so additional posterior instrumentation and fusion was performed.

The patients with radiculopathy showed improvement of symptoms at the final follow-up observation. In group 1, 9 of 10 cases showed the complete resolution of symptoms, and mild neuralgia was persistent in only 1 case, but it was substantially improved. In the 9 radiculopathy cases of group 2, the symptoms disappeared completely at the final follow-up observation. In regard to the patients associated with neurologic deficits at the time of diagnosis in group 1, there was one case of Frankel grade D (10\%), and it was improved to grade $\mathrm{E}$ at the time of the final follow-up observation. In group 2, a total of 4 cases $(25 \%)$ showed neurologic deficits and 2 cases with Frankel grade A were not changed even at the time of the final follow up observation. Nevertheless, the grade $\mathrm{B}$ and grade $\mathrm{C}$ patients were improved to grade $\mathrm{D}$ at the follow-up observation. One case each of both groups showed persistent mild posterior neck pain, but most cases showed substantial improvement, and the range of motion of the neck was recovered to the normal range, and so all the patients returned to work and their activities of daily living without restriction. In both groups, special complications did not develop during surgery or after surgery. Nevertheless, in 1 case of group 2, loosening of the screw to the anterior metal plate and the loss of reduction developed two weeks after surgery and so additional posterior surgery was performed.

\section{Discussion}

The incidence of a delayed diagnosis of a facet dislocation of the cervical spine with consequently delayed treatment was reported to be relatively high $(11-40 \%)$ [8,9]. As the common cause of a delayed diagnosis, the inability to undergo radiography because of an associated serious injury, insufficient clinical and neurological evaluation by doctors resulting in failing to perform radiography, patients refuse to visit medical institutions, patients who underwent radiography, but sufficient assessment of the lower cervical vertebrae was not done, and radiography was performed appropriately but an erroneous interpretation by clinicians were mentioned [10-14]. In our study, the cause of the delayed diagnosis of 5 cases out of 10 cases was that at the time of injury, radiography on the cervical vertebral area was taken, but the lower cervical area was not sufficiently examined and so the facet dislocation of the cervical spine was not diagnosed. In 3 cases the injury was shown on radiographs, but it was overlooked due to the carelessness of clinicians, and radiological tests on the cervical vertebra could not be performed in 2 cases because of unstable vital signs due to associate serious injury.

The most important points for diagnosing injury in the cervical vertebrae are to perform accurate and comprehen- 
sive assessment [2,15]. Kwon et al. [16] have reported that the cause of most delayed diagnoses is errors at the initial evaluation. Bohlman [17] have reported that approximately $1 / 3$ of serious cervical spine injuries are not initially detected. Therefore, Liu et al. [2] and Saddison et al. [15] stated that in alert patients, if cervical pain as well as direct tenderness are presented and even if neurological defects are absent, it is necessary to perform accurate cervical radiography. In the study conducted by Kahn et al. [3] for the patients with facet dislocation of the cervical spine, and this was diagnosed within 72 hours of injury, neurologic deficits were associated in $64 \%$ of the patients. On the other hand, in the group of patient with a diagnosis delayed for more than 72 hours, only $25 \%$ showed abnormal neurological diagnostic findings, and so the possibility of a delayed diagnosis is particularly higher for the patients without neurological deficit. In our study, although it was not statistically significant neurological deficit was detected in $25 \%$ of the patient group diagnosed immediately after admission and in $10 \%$ of the delayed diagnosis group, and a trend similar to the study reported by Kahn et al. [3] was shown. Therefore, it is thought that in patients without neurological symptoms or with mild symptoms, physicians are prone to overlook the injury and so comprehensive neurologic evaluation should be performed.

On the other hand, considering that in the study conducted by Platzer et al. [18], 8 cases of 18 delayed diagnosis cases $(44 \%)$ were patients for whom accurate neurological tests could not be performed and accurate physical examination could not be performed at the time of admission in many cases, and so it is thought that for patients admitted due to polytrauma, accurate radiological tests with keeping cervical spine injury in mind is an important factor. They suggested a method to evaluate the instability of injured areas for the unilateral facet fracture or subluxation cases by flexion and extension radiography under the supervision and observation of clinicians. We think that through such attempts the rate of overlooking injury and a delayed diagnosis may be reduced. In our study, similarly, for one case associated with multiple rib fractures and bilateral scapular fractures, radiating pain in the scapular area was misdiagnosed as pain caused by fractures, and so the diagnosis was delayed in that case. It has been reported that the evaluation of radiographs by experienced clinicians is required to prevent a delayed diagnosis caused by mistakes of clinicians at the time of the initial diagnosis [18]. It is thought that one way to reduce the rate of a delayed diagnosis is to perform computed tomography (CT) for the cases that are difficult to diagnose by simple radiography $[19,20]$. Although MRI can effectively differentiate injury involving soft tissue or intervertebral disc, nonetheless, as compared with CT, its sensitivity and specificity for fractures are not high [21], and so CT is thought to be more useful for the initial evaluation. Sekula et al. [22] proposed performing multidimensional CT together with plain lateral radiography for polytrauma patients to rule out injury in the cervical vertebral area, and the usefulness of CT for the evaluation of injury in the cervical vertebral area has been proven by several studies [20,23-26]. In our study, making the diagnosis using only simple radiography was difficult, and 1 case in group 1 (10\%) and 5 cases in group $2(33 \%)$ were diagnosed by CT. So it was found that CT was very useful for the diagnosis of unilateral facet dislocation of the lower cervical spine.

The diagnostic value of simple lateral radiography for prevertebral soft tissue swelling is well known. In our study, in the delayed diagnosis group, the degree of the soft tissue swelling at the inferior border of the 3rd cervical vertebral body was compared with that of the group diagnosed immediately after injury, and this was found to be significantly lower in the latter group. This suggests that in cases without neurological anomaly, the mild cases or the cases with little soft tissue swelling, the possibility of overlooking injury or a delayed diagnosis is relatively high. Therefore, it is thought that even if soft tissue swelling is not severe, patients should be evaluated with keeping the possibility of injury in mind.

It has been reported that reduction by skeletal traction is difficult when the diagnosis of facet dislocation of cervical vertebrae is delayed [27]. However, in our study, skeletal traction was performed on 8 of the 10 delayed diagnosis cases, and reduction was achieved prior to surgery in all cases. In addition, Kahn et al. [3] compared the success rate of reduction by cranial traction for the facet dislocation patients who were diagnosed early within 3 days with that of the patients whose diagnosis was delayed for more than 3 days. The early diagnosis group showed a $64 \%$ rate of successful reduction, but on the other hand, in the delayed diagnosis group, a $20 \%$ success rate was reported, and so reduction could be performed more readily if it is attempted within 3 days. We can speculate that their results are contradictory to our results because they did not distinguish unilateral facet dislocation from bilateral facet dislocation.

Liu et al. [2] examined a total of 9 cases of old fractures with distractive flexion injury stage 2 and 3, and they 
reported that it was difficult to anticipate reduction by skeletal traction, and so it was desirable to perform reduction by the posterior approach as well as anterior cervical discectomy and fusion, and fusion was achieved in all the cases. They reported that the postsurgical neurological condition of the 8 patients with neurological anomaly was substantially improved. Among the 4 cases with radiculopathy, hypoesthesia and motor weakness partially remained in 1 case and 3 cases recovered completely. They reported that in 4 cases with spinal cord injury, the Frankel grade was improved by 1 grade, the clinical symptoms were improved in almost all the patients and satisfactory results were obtained.

Hassan [4] has reported the results of surgical treatment of 12 cases of old fractures with stage 2 or 3 distractive flexion injury in the lower vertebra. It was observed that only 2 cases achieved reduction by skeletal traction, anterior and posterior surgery were performed on 9 cases, only posterior surgery was performed in 1 case and only anterior surgery was performed in 2 cases. They reported that all the patients showed improvement of their symptoms and neurological condition after surgery, and bone fusion was achieved in all cases.

However, in our study, for the patients with stage 1 and 2 distractive flexion injury, even if the diagnosis was delayed, reduction could be obtained by performing skeletal traction in all the cases. Consequently, without posterior surgery for open reduction, bone fusion was obtained by performing only anterior cervical discectomy and fusion in all the cases and clinically satisfactory results were obtained. It is thought that the stage 1 and 2 distractive flexion injuries are caused by relatively low energy and so reduction could be readily achieved by traction. In addition, the interval from injury to surgery was on average 27.6 days, and when it was compared with 2.8 months reported by Liu et al., reduction and surgery were preformed rapidly, and so the soft tissue factors that impede reduction were relatively less abundant.

\section{Conclusions}

The diagnosis of unilateral facet dislocation or subluxation caused by stage 1 or 2 distractive flexion injury is frequently delayed due to various reasons, and so considering the possibility of injury in the cervical area, careful neurologic examination and accurate radiologic evaluation should be done for the evaluation of trauma patients. The diagnosis using only simple radiography may be difficult in some cases, and so performing CT would be of help. Even for the cases with a delayed diagnosis, reduction could be obtained by skeletal traction prior to surgery, and thus performing only anterior cervical discectomy and fusion may be sufficient. Therefore, we think that reduction by skeletal traction should be performed as soon as unilateral facet dislocation or subluxation is diagnosed. In addition, even for the cases with a delayed diagnosis, the outcome of surgical treatments would not be greatly different from that of the cases that are diagnosed immediately after injury.

\section{REFERENCES}

1. Allen BL Jr, Ferguson RL, Lehmann TR, O'Brien RP. A mechanistic classification of closed, indirect fractures and dislocations of the lower cervical spine. Spine (Phila Pa 1976) 1982;7:1-27.

2. Liu P, Zhao J, Liu F, Liu M, Fan W. A novel operative approach for the treatment of old distractive flexion injuries of subaxial cervical spine. Spine (Phila $\mathrm{Pa}$ 1976) 2008;33:1459-64.

3. Kahn A, Leggon R, Lindsey RW. Cervical facet dislocation: management following delayed diagnosis. Orthopedics 1998;21:1089-91.

4. Hassan MG. Treatment of old dislocations of the lower cervical spine. Int Orthop 2002;26:263-7.

5. Korres DS, Nikiforidis P, Babis GC, Vlachou C, Lykomitros $\mathrm{V}$, Andreakos A. Old injuries of the lower cervical spine treated surgically. J Spinal Disord 1995;8:509-15.

6. Madhavan P, Monk J, Wilson-MacDonald J, Fairbank J. Instability due to unrecognised fracture-subluxations after apparently isolated injuries of the cervical spine. J Bone Joint Surg Br 2001;83:486-90.

7. Bartels RH, Donk R. Delayed management of traumatic bilateral cervical facet dislocation: surgical strategy. Report of three cases. J Neurosurg 2002;97(3 Suppl):362-5.

8. Bridwell KH, Lenke LG, McEnery KW, Baldus C, Blanke $\mathrm{K}$. Anterior fresh frozen structural allografts in the thoracic and lumbar spine. Do they work if combined with posterior fusion and instrumentation in adult patients with kyphosis or anterior column defects? Spine (Phila Pa 1976) 1995;20:1410-8.

9. Rorabeck CH, Rock MG, Hawkins RJ, Bourne RB. Unilateral facet dislocation of the cervical spine. An analysis of the results of treatment in 26 patients. Spine (Phila $\mathrm{Pa}$ 1976) $1987 ; 12: 23-7$. 
10. Reid DC, Henderson R, Saboe L, Miller JD. Etiology and clinical course of missed spine fractures. J Trauma 1987;27:980-6.

11. Gerrelts BD, Petersen EU, Mabry J, Petersen SR. Delayed diagnosis of cervical spine injuries. J Trauma 1991;31: 1622-6.

12. Davis JW, Phreaner DL, Hoyt DB, Mackersie RC. The etiology of missed cervical spine injuries. J Trauma 1993;34:342-6.

13. Alker GJ, Oh YS, Leslie EV, Lehotay J, Panaro VA, Eschner EG. Postmortem radiology of head neck injuries in fatal traffic accidents. Radiology 1975;114:611-7.

14. MacDonald RL, Schwartz ML, Mirich D, Sharkey PW, Nelson WR. Diagnosis of cervical spine injury in motor vehicle crash victims: how many X-rays are enough? J Trauma 1990;30:392-7.

15. Saddison D, Vanek VW, Racanelli JL. Clinical indications for cervical spine radiographs in alert trauma patients. Am Surg 1991;57:366-9.

16. Kwon BK, Vaccaro AR, Grauer JN, Fisher CG, Dvorak MF. Subaxial cervical spine trauma. J Am Acad Orthop Surg 2006;14:78-89.

17. Bohlman HH. Acute fractures and dislocations of the cervical spine: an analysis of three hundred hospitalized patients and review of the literature. J Bone Joint Surg Am 1979;61:1119-42.

18. Platzer P, Hauswirth N, Jaindl M, Chatwani S, Vecsei V, Gaebler C. Delayed or missed diagnosis of cervical spine injuries. J Trauma 2006;61:150-5.

19. LeBlang SD, Nuñez DB Jr. Helical CT of cervical spine and soft tissue injuries of the neck. Radiol Clin North Am 1999;37:515-32, v-vi.

20. Rethnam U, Yesupalan RS, Bastawrous SS. The Swimmer's view: does it really show what it is supposed to show? A retrospective study. BMC Med Imaging 2008;8:2.

21. Klein GR, Vaccaro AR, Albert TJ, et al. Efficacy of magnetic resonance imaging in the evaluation of posterior cervical spine fractures. Spine (Phila Pa 1976) 1999;24:771-4.

22. Sekula RF Jr, Daffner RH, Quigley MR, et al. Exclusion of cervical spine instability in patients with blunt trauma with normal multidetector CT (MDCT) and radiography. Br J Neurosurg 2008;22:669-74.

23. Dailey AT, Shaffrey CI, Rampersaud R, et al. Utility of helical computed tomography in differentiating unilateral and bilateral facet dislocations. J Spinal Cord Med 2009;32:43-8.

24. Chevrot A, Drape JL, Godefroy D, Dupont AM. Imaging of the painful cervical spine. J Radiol 2003;84(2 Pt 2):181239.

25. Mink JH, Gordon RE, Deutsch AL. The cervical spine: radiologist's perspective. Phys Med Rehabil Clin N Am 2003; 14:493-548, vi

26. Shanmuganathan K, Mirvis SE, Levine AM. Rotational injury of cervical facets: CT analysis of fracture patterns with implications for management and neurologic outcome. AJR Am J Roentgenol 1994;163:1165-9.

27. Cotler HB, Miller LS, DeLucia FA, Cotler JM, Davne SH. Closed reduction of cervical spine dislocations. Clin Orthop Relat Res 1987;(214):185-99. 